\title{
THERMALLY ASSISTED ION BEAM ETCHING OF POLYTETRAFLUOROETHYLENE A NEW TECHNIQUE FOR HIGH ASPECT RATIO ETCHING OF MEMS
}

\author{
Erwin Berenschot, Henri Jansen, Gert-Jan Burger, Han Gardeniers, and Miko Elwenspoek
}

MESA Research Institute, University of Twente, P.O. Box 217, 7500AE Enschede, The Netherlands

Phone: X-31-53-892640; Secr.: X-31-53-892751; Fax: X-31 53-309547; E-mail: H.V.Jansen@eltn.utwente.nl

\begin{abstract}
In micromechanics, the etching of high aspect ratio structures in polymers is a prime technology. Normally, oxygen-based reactive ion etching or the LIGA technique are used to achieve this goal. This paper reintroduces a different idea to create deep trenches at high etch speed: The ion beam etching of Teflon ${ }^{\mathrm{TM}}$. Because of its extraordinary properties the etch selectivity with respect to most other materials is over 1000. A model is proposed to explain the high etch rate and selectivity. Generally, the etching ions are highly energetic and material from different sources is sputtered on top of the sample. The high selectivity, high anisotropy, and sputtering of material are thought to be responsible for the forming of micrograss during etching a sample. Ways are given to decrease or increase this grass. The high potential of this technique will be discussed and applications will be shown. Especially, the use of etched Teflon ${ }^{\mathrm{TM}}$ for direct moulding is believed to become the main use of this technique.
\end{abstract}

\section{INTRODUCTION}

The sputtering of fluorocarbon (FC) polymers is of significant industrial interest. A special member of this family is the linear, quasi-crystalline polymer polytetrafluoroethylene (PTFE) better known under its trademark Teflon ${ }^{\mathrm{TM}}$. Stringent application requirements concerning friction, adhesion, dielectric properties, hydrophobicity, chemical inertness, and high temperature survivability have fostered the widespread utilisation of FC polymers. Similarly, there are many applications in which a thin coating or adhesively bonded FC laminate would be of great utility [1].

This paper reviews FC polymer vacuum sputtering technologies from which the ion beam etching (IBE) of PTFE seems to be the most promising [1]. Although laborious work concerning the IBE of PTFE was already performed by NASA in the mid seventies, to our knowledge there has been no study dealing with the lithographical patterning of PTFE for MEMS applications. Most investigators were mainly interested in increasing grasslike roughness on the PTFE surface to be able to attach it to other materials. However, for moulding purposes and for most other micromechanical uses, the grass is highly unwanted.

The review is followed by an experimental investigation of the thermally assisted ion beam etching (TAIBE) of PTFE. The etch apparatus is schematically shown and it is tried to explain the etch mechanism of this technique. After this, the difference in etch rate with respect to other materials and the forming of grass is treated. After the experimental set-up and the results, some applications will be shown.

Because of the misleading names for many well-known vacuum process technologies, sometimes important mechanisms are not recognised. Therefore, this paper will start with a first attempt to order all the various vacuum process technologies more consistently.

\section{NOMENCLATURE}

To etch polymers it is possible to use the energy content of any kind of particles, such as $i)$ neutrals $(N)$ : sand blasting (NBE), ii) radicals $(\boldsymbol{R})$ : ozone stripping or plasma/ radical etching (RE), iii) ions (I): ion beam etching (IBE), iv) electrons (E): electron-beam evaporation/etching (EBE), v) phonons (T): thermal decomposition/etching (TE), or vi) photons (P): laser ablation i.e. photon beam etching (PBE). These energetic particles are readily produced in a plasma environment and generally they will interfere with each other synergetically, while increasing the etch rate enormously, as in chemical assisted ion beam etching (CAIBE) or reactive ion etching ("RIE") [2].

In literature, the given sputter techniques are not always labelled consistently. Therefore, the next labelling is introduced. The labelling ends always with the main purpose of the technique; etching (E), deposition (D), or modifying (M). If the e.g. etching is made possible by a collimated stream of particles, the technique will end with "beam etching" (BE). In front of this the type of energetic particle is placed and if focused the label is extended with the letter " $F$ ". So, focused ion beam etching (FIBE) stands for a focused stream of ions which are etching a substrate. Similarly, the focused electron beam resist technique is labelled with "FEBM". If there is no stream of etching particles the labelling only ends with "etching" (E). So, ozone stripping is an example of "radical etching" (RE). When the (stream of) particles is accompanied by a different source of (a stream of) particles, the labelling is extended with "assisted" (A) and if the flux of particles have more than one energy reservoir it is extended with this source. Thus, chemical assisted reactive ion beam etching (CARIBE) stands for a stream of reactive ions assisted by a flux of chemicals.

The plasma etching of polymers is normally fulfilled at elevated temperatures (e.g. $150^{\circ} \mathrm{C}$ ), so a better name would be "thermally assisted radical etching" (TARE). The term "reactive ion etching" (RIE) is a misleading name. As for the beam etching techniques it should be depending on the type of energetic particles and substrate material which label it should deserve. In most RIE cases a directed ion flux is accompanied by an isotropic radical flux. So, a more correct name would be "reactivily assisted ion beam etching" (RAIBE). 


\section{REVIEW OF VACUUM FC ETCHING}

Already in 1978 Banks et al. reviewed several FC polymer sputtering techniques including thermal, plasma, and ion beam sputtering. Ion beam etch rates (ER) up to $2 \mathrm{~mm} / \mathrm{hr}$ for PTFE were determined and shown to depend predominantly upon the ion beam power flux: $E R=\mathrm{P}^{1.4}$, where $\mathrm{P}$ is the power flux in $\mathrm{W} / \mathrm{cm}^{2}[1,12,13]$. High peel strengths were measured for epoxy bonds to various ion beam sputtered $\mathrm{FC}$ polymers and appear to be related to the surface microstructures (grass) resulting from IBE. Polychlorotrifluoroethylene (PCTFE), fluorinated ethylene propylene (FEP), perfluoroalkoxy (PFA) Teflon ${ }^{\mathrm{TM}}$, Tefsel ${ }^{\mathrm{TM}}$, and polyoxymethylene (POM) gave identical results $[1,3,12]$.

Jansen et al. treated a few other FC deposition (spincoating) and sputter (focused electron beam and plasma) techniques. The thin-film properties were comparable with those of commercially available bulk PTFE [4]. In order to track the chemical nature of the deposited FC thin-film, in ref. [5] the authors presented the results of X-ray photoelectron spectroscopy (XPS) analyses.

To be able to control the etched profile -especially steep walls are useful- the particles are collimated and directed to the surface to be etched. This can be achieved by parabolic mirrors (photons), wind tunnels (neutrals, radicals), or electrical fields (ions, electrons). In fact, the PBE and IBE equipment are typical examples of this technique.

During etching deep trenches into substrate materials specific problems may occur which will hamper the aspect ratio ( $A R=$ depth/width) of the trench such as the depletion of etching particles due to diffusion lag. Until now, many techniques have been developed in order to achieve high $\mathrm{AR}$ structures in polymers: photolithography such as LIGA (PBM), reactive ion etching (RAIBE), thermally assisted ion/electron beam etching (TAIBE or TAEBE), and others.

PBM: A direct way is the use of conventional photolithography. However, because of the rather high wavelength of the exposing source and the limited thickness of the e.g. spin-coated photo sensitive polymer, the AR of the structures are seldom exceeding 7 and it is difficult to create submicron features [6]. To increase the aspect ratio short wavelength directional $\mathrm{x}$-rays from a cyclotron source are used. However, this so-called LIGA technique is expensive [7].

RAIBE: In order to extend the limit of PBM special three level "RIE" techniques have been developed [8]. Electron sensitive polymers are used in the first level to create submicron structures (EBM) [9]. Nevertheless, the AR is usually not higher than 10 because of the relatively high operating pressures during copying the pattern with RIE into the third level. To increase the AR low pressure, high density RIE machines are developed, but these machines are costly [10].

TAIBE: IBE is a simple technique to create high $A R$ trenches into PTFE. The technique was demonstrated first by NASA [11-13] in the mid seventies. It uses the extraordinary properties of PTFE e.g. its extremely high melt viscosity [4]. Because of this, PTFE preserves its shape even at temperatures near its melting point. Therefore IBE can be used at elevated temperatures to achieve high etch rates up to several tens of microns per minute. The most straightforward name for this type of etching equipment is thermally assisted ion beam etching (TAIBE). The selectivity with respect to the mask typically exceeds 1000 . Because of the low operating pressure during IBE the AR is superior to RIE.

TAEBE: The use of electrons to etch a material is found in the e-beam evaporator. Energetic electrons are directed to a material what will melt and evaporate (FEBE). As for TAIBE highly directional profiles are created in PTFE. In stead of the focused electron beam of an evaporator, a broad band electron source can be generated with the help of an IBE. Electrons from a current heated tungsten wire are directed to the substrate with the help of a grid at a positive potential with respect to the wire. A logical name for such a system would be electron beam etcher (EBE) and at elevated temperatures the process becomes thermally assisted electron beam etching (TAEBE). The lower operating pressure opens the possibility to achieve even higher aspect ratios as in the case of TAIBE.

TAPBE: It is straightforward to use a laser or a halogen lamp, assisted with a heat source, to etch a substrate (i.e. TAFPBE and TAPBE respectively). A $1200 \mathrm{~nm}$ photon has an energy content of $1 \mathrm{eV}$, so polymers -especially PTFE- are easily sputtered at elevated temperatures. To be able to create submicron structures it is necessary to use photons with a small wavelength like $\mathrm{x}$-rays and special $\mathrm{x}$-ray-opaque materials are needed to transfer a pattern.

\section{ETCH APPARATUS}

In figure 1 a simplified schematic of the IBE apparatus which is used in this study is shown. It consist of four independent parts: i) The vacuum system, ii) the ion source, iii) the substrate holder, and iv) the heater.

The vacuum system: To direct a beam of atomic particles over a large distance $(>5 \mathrm{~cm})$ it is necessary to create a pressure below $1 \mathrm{~m}$ Torr. This is achieved by mounting a turbopump in series with a rotary pump to the reactor which also removes reaction products.

The ion source: The energetic particle flux is generated with the help of an electron bombardment ion source (Kaufman type). Inert (Ar) or reactive $\left(\mathrm{O}_{2}\right)$ gases are let into the small chamber $(l=42 \mathrm{~mm}$ and $\varnothing=33 \mathrm{~mm})$. To create a plasma, a current is forced through a tungsten wire which gives a cloud of electrons surrounding the wire i.e. the cathode. These electrons are forced to the walls of the chamber which have a positive potential i.e. the anode. In their way to the walls the electrons collide with the gases creating all kinds of energetic particles including ions and electrons. Due to the increasing amount of charged particles a plasma is started. To increase the efficiency of the electrons in creating plasma particles, magnets are surrounding the Kaufman source in a way that the electrons are describing a spiral like path to the anode. So, their lifetime is increased and therefore the particle generation. Beam extraction is accomplished by a flat, two-grid carbon ion optics system. A neutraliser is used to prevent i) beam diffraction, ii) charging of the substrate, and iii) electrical breakdown of electronic circuits in the substrate when turning the beam of.

The substrate holder: To be able to control the etched profile the system features substrate rotation with an adjustable angle and water cooling at $18^{\circ} \mathrm{C}$.

The heater: To control the temperature of the substrate, it is mounted on top of a 150 Watt Joule heater or a 150 Watt Halogen lamp with a $50 \mathrm{~cm}$ spot size is used. 


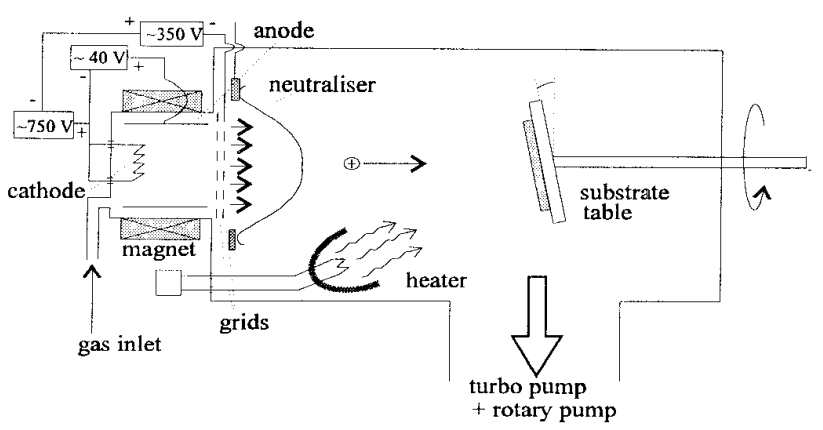

Figure 1: Basic schematic for thermally assisted IBE.

\section{ETCH MECHANISM}

Heating of PTFE in the range $360^{\circ} \mathrm{C}$ to $600^{\circ} \mathrm{C}$ caused thermal decomposition involving formation of free radicals due to random polymer chain scission. It left a carbon residue and the largest scission fragment was $\mathrm{C}_{3} \mathrm{~F}_{8}$, with $\mathrm{C}_{2} \mathrm{~F}_{4}$ as the primary product $[14,15]$. The plasma decomposition of PTFE at 11.5 Torr resulted in a similar distribution of reaction products probably due to the short mean free path " $\lambda$ " and further fragmentation in the plasma glow [15]. However, the low pressure environment in IBE $(<0.2 \mathrm{mTorr})$ allows large scission fragments $\left(>\mathrm{C}_{3} \mathrm{~F}_{8}\right)$ to escape from the substrate with minor carbon residue. This would explain the quasicrystallinity of the ion beam sputter deposited FC thin-films. This in contradiction to the other techniques which produce amorphous highly crosslinked FC thin-films [1].

Hall and Green [16] and Rost [17] measured the IBE etch rates of FEP and PTFE respectively and found them to be approximately two orders of magnitude higher than those of metals and glass ( 100 CF2 groups per incident ion). Rost attributed the higher etch rates of PTFE to break-up of strong bonds in molecular chains producing a wide distribution of scission fragments that leave the target surface by thermal effects where only small intermolecular forces must be overcome. Hall and Green also measured erosion rates of similar linear addition polymers i.e. polyvinylfluoride and the linear polypropylene but did not observe elevated erosion rates. More rapid crosslinking than depolymerisation was felt to cause this result. The FEP sputter yields exhibited a significant sensitivity to ion current density. This is a unique characteristic in that most materials have sputter yields nearly independent of current density [18-20]. Especially this last result is indicative for local heating as an important factor in the IBE of PTFE.

As already stated the etch rate of PTFE is highly temperature dependent. The sample can be heated e.g. from the backside with the help of a Joule heater or from the front side by way of a lamp or incoming ion flux. Especially the ions carry with them a large amount of kinetic energy (typically $1000 \mathrm{eV}$ per incident ion). The heat at the front side is partly leaving the surface as evaporation/kinetic energy of etch products and black body radiation. The rest will travel trough the PTFE bulk to the back side, which can be water cooled at $18^{\circ} \mathrm{C}$ or Joule heated at a fixed temperature $T_{b}$. Together with the heat resistance, this flow of heat will create a temperature gradient. It is assumed that the temperature distribution in the PTFE is not changing in time: In other words the -distributed- heat capacity is fully charged. In the same way it is assumed that the surrounding is at a stable temperature $T_{S}$. Writing the energy balance for the front side of the PTFE sheet we find at thermodynamical equilibrium:

$$
\begin{gathered}
\mathrm{Q}_{\text {ion }}+\mathrm{Q}_{\text {photon }}=\mathrm{Q}_{\text {conduction }}+\mathrm{Q}_{\text {radiation }}+\mathrm{Q}_{\text {evaporation }} \\
\mathrm{U}_{\mathrm{i}} \mathrm{J}_{\mathrm{i}}+\eta \varepsilon \mathrm{U}_{\mathrm{p}} \mathrm{I}_{\mathrm{p}} / \mathrm{A}=\lambda\left(\mathrm{T}_{\mathrm{f}} \mathrm{T}_{\mathrm{b}}\right) / t+\varepsilon \sigma\left(\mathrm{T}_{\mathrm{f}}{ }^{4}-\mathrm{T}_{\mathrm{s}}{ }^{4}\right)+\mathrm{ER}(\Delta \mathrm{p}) \Delta \mathrm{H}^{\mathrm{O}}+\mathrm{Q}_{\mathrm{kin}}
\end{gathered}
$$

With $U_{i}[V]$ the acceleration voltage of the ions, $J_{i}\left[A / m^{2}\right]$ the ion current density, $\eta$ the efficiency of the lamp in heating the sample with photons, $\varepsilon$ the emissivity depending on material properties, $U_{p}[V]$ the voltage over the lamp, $I_{p}[A]$ the current through the lamp, A $\left[\mathrm{m}^{2}\right]$ the spot size, $\lambda=0.24$ $[\mathrm{W} / \mathrm{mK}]$ the specific heat conductance of PTFE, $\mathrm{T}_{f}, \mathrm{~T}_{\mathrm{b}}$, and $\mathrm{T}_{\mathrm{S}}$ the temperature $[\mathrm{K}]$ of the front, back, and surrounding surface respectively, $\mathrm{t}[\mathrm{m}]$ the thickness of the sample between $\mathrm{T}_{\mathrm{f}}$ and $\mathrm{T}_{\mathrm{b}}, \sigma=5.7 * 10^{-8}\left[\mathrm{~W} / \mathrm{m}^{2} \mathrm{~K}^{4}\right]$ the StefanBoltzmann constant, and ER $\left[\mathrm{mol} / \mathrm{m}^{2} \mathrm{~s}\right]$ is the etch rate which is a function of the pressure difference $\Delta p$ between the vapour pressure $p_{V}$ of the PTFE and the partial pressure $p_{p}$ in the vacuum system. At high etch rates or sample temperature $\Delta p \approx$ $p_{V}$ which is a function of the temperature $T_{f} \cdot \Delta H^{0}=\Delta H^{0}$ sub $=\Delta$ $\mathrm{H}^{\mathrm{O}}$ fus $+\Delta \mathrm{H}^{\mathrm{O}}$ vap $[\mathrm{J} / \mathrm{mol}]$ is the molar enthalpy (of sublimation =fusion+vaporation) at 1 atm i.e. the energy needed to bring one mole of solid PTFE into the vapour phase. This figure is assumed to be independent of temperature in the range we are working (i.e. 20 to 300 degrees Celcius). $Q_{k i n}\left[W / \mathrm{m}^{2}\right]$ is the heat flux due to kinetic energy. Because the amount of particles removed per incident ion (i.e. the yield) is so high and not depending on the ion energy or ion current flux alone but rather to the power flux it is thought that $Q_{\text {kin }}$ can be neglected.

Photon source: To find the efficiency of a halogen photon source of $\mathrm{P}=150 \mathrm{~W}$ to heat a sample, three different materials with the same dimensions $\left(10 \times 10 \times 1 \mathrm{~cm}^{3}\right)$ were placed into the vacuum directly in front of the photon source: aluminium, oxidised steel, and PTFE. The sample was mounted with thin plastic wires to ensure a bad thermal contact with the surrounding. Indeed, after turning off the lamp, the temperature of the samples of steel and aluminium at $45^{\circ} \mathrm{C}$ didn't change for more than one degree over 1 hour, indicating that there is little heat transported due to conduction or radiation. After creating a vacuum of $10^{-6}$ Torr the sample was heated for a period of time while monitoring the sample temperature $\Delta \mathrm{T}[\mathrm{K}]$ with a thermocouple. The heating is stopped when a certain temperature rise $\left(\Delta T=15^{\circ} \mathrm{C}\right)$ is achieved and the time is clocked. Now, we are able to calculate the efficiency with the help of the formula: $\eta=\mathrm{cm} \Delta$ $\mathrm{T} / \varepsilon \mathrm{Pt}$, with $\mathrm{c}[\mathrm{J} / \mathrm{gK}]$ the specific heat capacity of the sample and $\mathrm{m}[\mathrm{g}]$ its mass as found in table 1. For example, to increase the temperature of steel 19 degrees 3 minutes photon heating was needed and we calculate $\eta=0.2$. So, only 30 Watts are used for heating the sample! Nevertheless, the incoming heat flux is still $0.6 \mathrm{~W} / \mathrm{cm}^{2}$. As can be concluded from table 1, the efficiency of the lamp is 0.2 , when aluminium or steel are taken as a reference but 0.04 for the PTFE sample. This is unlikely because the photon source was placed in the same way with respect to the sample. It is known that a difference in roughness for the same material is responsible for a major change in emissivity. So, when we assume that the emissivity $\varepsilon=0.2$ of PTFE than we calculate 


\begin{tabular}{|l|l|l|l|l|l|}
\hline material & $\mathrm{m}[\mathrm{g}]$ & $\mathrm{c}[\mathrm{J} / \mathrm{gK}]$ & $\varepsilon$ & $\mathrm{t}[\mathrm{s}]$ & $\eta$ \\
\hline aluminium & 168 & 0.88 & 0.24 & 420 & 0.2 \\
\hline steel & 484 & 0.48 & 0.80 & 180 & 0.2 \\
\hline PTFE & 147 & 1.05 & 0.9 & 420 & 0.04 \\
\hline
\end{tabular}

Table 1: Data to find the efficiency of the photon heater.

$\eta=0.2$. It is also thinkable that the PTFE is loosing material i.e. energy during the heating of the photon source. So, little of the absorbed energy is used to increase the heat in the heat capacity.

In principle, the photon source is a black body radiator with an equivalent temperature $T_{p}=\left(\eta U_{p} I_{p} / \sigma A\right)^{1 / 4}=500 \mathrm{~K}$. However, because it is driven by a remote power source it is treated separately. Nevertheless, when the lamp is included in the radiation term then $Q_{\mathrm{rad}}=\varepsilon \sigma\left(\mathrm{T}_{\mathrm{f}}{ }^{4}-\mathrm{T}_{\mathrm{s}}{ }^{4}-\mathrm{T}_{\mathrm{p}}{ }^{4}\right)$.

Note that the cathode and the neutraliser are also radiating photons to the sample. This energy input should be added in the same way as already done.

Conduction: Let's take a typical IBE setting of $1000 \mathrm{eV}$ at $1 \mathrm{~mA} / \mathrm{cm}^{2}$ i.e. $Q_{\text {ion }}=1 \mathrm{~W} / \mathrm{cm}^{2}$. If the only lost of heat was due to conduction and $t=3 \mathrm{~mm}$ we are able to calculate the temperature gradient: $T_{f}-T_{b}=Q_{i o n} t / \lambda=120 K$. This is a major temperature rise and can be even higher when the thermal contact of the PTFE sample is incorrect.

Sovey found that the etch rate increased from 180 to $640 \mu \mathrm{m} / \mathrm{hr}$ as the target reference temperature increased from $200^{\circ} \mathrm{C}$ to $280^{\circ} \mathrm{C}$ [12]. At $280^{\circ} \mathrm{C}$ condition, photomicrographs indicated evidence of local melting and spire like structures no longer existed. The ion etch rates tended to asymptote to approximately $100 \mu \mathrm{m} / \mathrm{hr}$ at target temperatures below $150^{\circ} \mathrm{C}$. They concluded: "This implies that physical sputtering probably dominates over thermal processes at target reference temperatures less than $150^{\circ} \mathrm{C} . "$. However, at the fixed parameters Sovey used $\left(750 \mathrm{eV}, 0.5 \mathrm{~mA} / \mathrm{cm}^{2} \Rightarrow 375 \mathrm{~mW} / \mathrm{cm}^{2}\right.$ and a $3 \mathrm{~mm}$ thick PTFE sample) we are able to calculate a possible temperature rise of $45^{\circ} \mathrm{C}$ because of the ion heat flux. Indeed, $280^{\circ} \mathrm{C}+45^{\circ} \mathrm{C}=325^{\circ} \mathrm{C}$ is close to the melting point of PTFE at atmospheric pressures $\left(327^{\circ} \mathrm{C}\right)$. The local pressure might be 1 atm due to the high etch rate.

Radiation: To find out the importance of the black body radiation we assume $\varepsilon=0.9$ and the sample to be at $600 \mathrm{~K}$ i.e. the temperature of melting for PTFE: $\mathrm{Q}_{\mathrm{rad}}=\varepsilon \sigma\left(\mathrm{T}_{\mathrm{f}}{ }^{4}-\mathrm{T}_{\mathrm{S}}{ }^{4}\right)=0.6$ $\left[\mathrm{W} / \mathrm{cm}^{2}\right]$. Again an important drain of energy.

Sublimation: To calculate the heat flux due to sublimation it would be convenient to know how the pTphase diagram looks like. This plot contains the triple point which is characteristic for a certain material and represents the only pressure and temperature at which the vapour-, liquid, and solid of a specific material can coexist in equilibrium under the pressure of the vapour alone. For example, in figure $2 \mathrm{a}$ the pT-phase diagram of pure water is given. Its triple point is located at $0.0098^{\circ} \mathrm{C}$ and 4.5 Torr. At pressures lower than this point only vapour and solid can coexist in equilibrium under the pressure of the vapour alone as represented by the sublimation line. This line connects a certain temperature $\mathrm{T}_{\mathrm{f}}$ with a single pressure: the vapour pressure $p_{\mathrm{v}}$. The vapour pressure is increasing exponentially with the sublimation temperature:

$$
p_{V}\left(T_{f}\right)=p^{o} \exp \left(\left(T_{f} \Delta S^{o}-\Delta H^{o}\right) / R T_{f}\right)
$$

With $\mathrm{R}=8.3[\mathrm{~J} / \mathrm{Kmol}]$ the universal gas constant, $\Delta \mathrm{S}^{\mathrm{O}}$ the molar entropy at $1 \mathrm{~atm}$, and $\mathrm{p}^{\mathrm{O}}=1 \mathrm{~atm}$ the reference pressure where $\Delta \mathrm{G}^{\mathrm{O}}=\Delta \mathrm{H}^{\mathrm{O}}-\mathrm{T}_{\mathrm{f}} \Delta \mathrm{S}^{\mathrm{O}}=0 \Rightarrow \mathrm{T}_{\mathrm{f}}=\mathrm{T}^{\mathrm{O}}=\Delta \mathrm{H}^{\mathrm{O}} / \Delta \mathrm{S}^{\mathrm{O}}$. So, by definition $\mathrm{T}^{\mathrm{O}}$ is the temperature (usually the boiling temperature $T_{b}$ ) where $p_{v}=p^{0}$. In equilibrium, the amount of solid going into the vapour phase $\left[\mathrm{mol} / \mathrm{m}^{2} \mathrm{~s}\right]$ equals the amount of vapour going into solid at a rate [47]:

$$
E R\left(p_{V}\right)=p_{V} N_{A}\left(2 \pi m k T_{f}\right)^{-1 / 2}
$$

With $\mathrm{N}_{\mathrm{A}}=6 * 10^{23}\left[\mathrm{~mol}^{-1}\right]$ the number of Avogadro, $\mathrm{m}[\mathrm{kg}]$ the mass of the molecule, and $\mathrm{k}=14 * 10^{-24}[\mathrm{~J} / \mathrm{K}]$ the constant of Boltzmann. Now, when a piece of ice is placed into vacuum the vapour is removed (turbo) or frozen to cold surfaces (cryo) and the back streaming is made impossible. It is reasonable to assume that the sublimation is going on with the same rate, thus the ice is losing mass presuming the pump can handle the amount of vapour which is produced. The rate of disappearance is low, of course, because the vapour pressure is low. To give an indication; assume the vapour pressure is $10^{-6}$ [Torr] i.e. $133^{*} 10^{-6}[\mathrm{~Pa}]$ at a temperature of $1000[\mathrm{~K}]$, the interatomic distance is $3[\AA]$, and the mass of the molecule is $33^{*} 1.6^{*} 10^{-27}[\mathrm{~kg}]$. Then the rate of sublimation is approximately $3 * 10^{-6} \quad\left[\mathrm{~mol} / \mathrm{m}^{2} \mathrm{~s}\right] \approx 0.2$ $[$ monolayers $/ \mathrm{s}] \approx 0.6[\AA / \mathrm{s}]$. Note: Every vacuum system removes gases selective. For example, a turbopump removes water vapour much easier than hydrogen gas. This means that the water vapour pressure is lower than indicated by the pressure sensor of the vacuum system. Nevertheless, the vapour pressure is not always low and increases exponentially with temperature. So, a different material can be removed quite fast when it is heated sufficiently. When the sample is heated and the reactor walls are at room temperature the walls will act as an adsorption pump and the etch rate is completely determined by the vapour pressure of the sample.

Now, let's take a PTFE sample. PTFE consists of many different sized FC chains. Therefore, the pT-plot of PTFE will not have single equilibrium lines but a broad spectrum and not a triple point but a triple line. During heating PTFE first the short chains will sublimate (or melt and evaporate) leaving the longer chains in the solid. Increasing the temperature more and more the longest chains will evaporate at last. It would be quite interesting to see how the perfluoroalkanes $\left(\mathrm{C}_{n} \mathrm{~F}_{2 n+2}\right.$ like $\mathrm{C}_{10} \mathrm{~F}_{22}$ ) behave under the same conditions as PTFE, because PTFE is believed to consist of such oligomers [48]. Because these molecules have a unique chain length their pTplot will be quite normal and we should be able to calculate the ER of such materials with the help of the theory presented in this paper.

Note that -in principle- TABE opens a whole scale of possibilities to pattern an arbitrary substrate material. For example, zinc could be IBE patterned with a high selectivity if we reach a vacuum low enough for its triple point (otherwise it would first melt and then vaporise i.e. it would become isotropic) together with substrate heating [47]. As a mask, a material with a much higher melting point or a cooled (zinc) shadow mask could serve. 

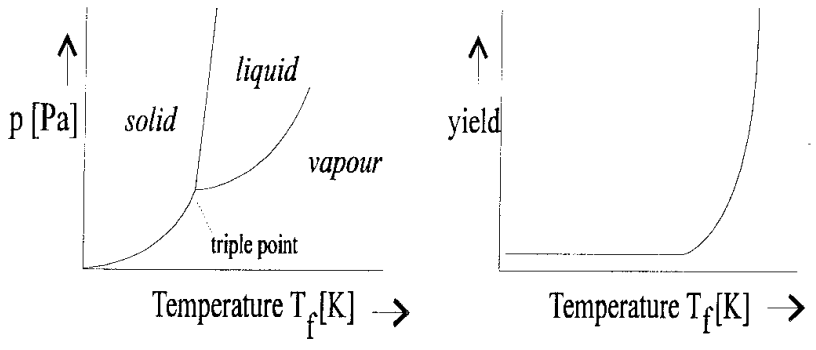

Figure 2: a) pT-diagram and b) temperature dependency of the sputter yield.

Any way, the low thermal conductivity together with the comparable high vapour pressure at moderate temperatures might account for the difference in etch rate between PTFE and inorganic materials. In figure $2 \mathrm{~b}$ a plot is shown giving the yield of an incident ion as function of the temperature. Evidently, the yield of an incoming ion is increasing with temperature because particles are leaving the solid at a higher rate than particles coming back. At relatively low sample temperatures the etch rate is determined by the pumping speed of the vacuum system because $\Delta p=p_{v}-p_{p} \neq p_{v}$. and the yield of an ion will be between 1 and 4 for most materials The energetic ion is removing just a few atoms due to its impuls. However, when the temperature of the sample is much higher than the surrounding surfaces the IBE system will act as an adsorption pump and all the PTFE material is deposited at the reactor walls. In these cases the etch rate is determined by the vapour pressure and the yield can be over 100. The TAIBE of PTFE is enormous anisotropic which might be linked with its high melt viscosity. But why does PTFE possess such a high anisotropy /melt viscosity? It is possible that the longer chains in the PTFE, which are melting at much higher temperatures than the smaller chains, are acting as the framework of the PTFE. So, it preserves the shape of the PTFE in the regions where ion bombardment fails. However, in the open regions after the smaller chains have left, the longer chains can be broken by the ions and the products can leave the surface. The only question left is: Why do other comparable polymers not etch at increased speed? The answer might be found in the way they degrade at elevated temperatures. For example, polypropylene would leave a carbonic residue which prevents further high speed etching.

\section{GRASS}

Large cone or grasslike surface microstructures, with diameters of a few microns and lengths up to more than 100 micron, resulting from ion or if bombardment have been observed for FEP and PTFE substrates [16,21]. Figure 3 shows a typical example. The structures are found to be always parallel to the direction of the incident ions.

IBE guarantees an excellent anisotropy because of its highly directional ion flux and low operating pressure. Due to the high selectivity of PTFE with respect to other materials, micromasking will cause the grass problems which are identical with high anisotropic RIE trench etching $[22,44]$. To overcome this problem substrate rotation can be used. Also, the substrate normal should be tilted with respect to the ion flux direction. This solution is visualised with the help of figure $4 a / b$.

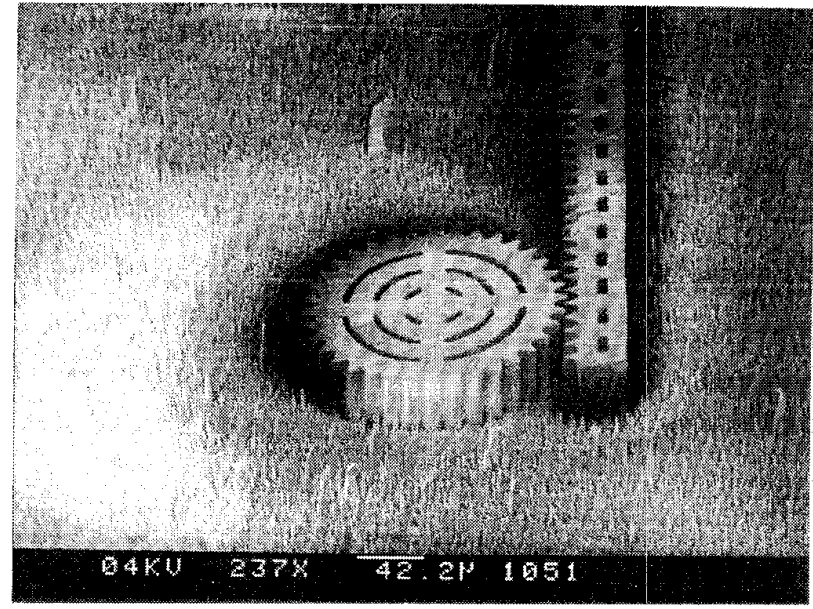

Figure 3: Forming of grass during IBE of PTFE.

However, care should be taken while using substrate rotation as demonstrated with the help of figure $4 \mathrm{c}$. In this figure the depth profile of a pinhole in the mask design is shown. Because of the tilted substrate rotation, a conelike structure will develop directly under the pinhole position and IBE grass in these trenches can't be prevented because of the highly directional ion flux. Moreover, due to geometrical reasons the etch rate in depth will decrease inversely proportional in time.

Another way to prevent the forming of grass is preventing non-PTFE particles to deposit on top of the PTFE surface as shown in ref.45. The authors found that as much as $5 \frac{1}{2}{ }^{*} 10^{-4}$ particles from miscellaneous sources per incident ion were sputtered on the sample.

Mask particles: The first contamination of particles is due to sputtered mask material. This deposit can be lowered by decreasing the ion energy. However, the ion current density must be increased simultaneously to ensure a certain etch rate. A different solution is the use of a cooled PTFE shadow mask. At the same time the PTFE sample should be heated to increase the selectivity between mask and sample.

Reactor particles: A major source of particles is due to sputtered reactor material. During the IBE of PTFE we found that stainless steel from the sample holder was deposited at the sidewalls of the PTFE trenches. This type of grass was prevented by using a PTFE sample holder.

Grid particles: The next important particle source is the sputtering of the second grid of the carbon ion optics system. After passing the first grid, the ions are accelerated to the second grid at e.g. $-1000 \mathrm{~V}$. When the grids are misaligned, the second grid is hit by the ions and will sputter carbon particles on top of the PTFE sample. Accurate alignment and making the holes in the second grid bigger than those in the first grid decreases this type of micromasking. Note that this solution increases beam diffraction. At this moment, effort is put into manufacturing a micromachined self-aligned double grid ion optics system.

Neutraliser particles: The next possible source of particles is the tungsten neutraliser which is in front of the ion beam to prevent beam diffraction. We didn't notice beam diffraction so we removed the neutraliser completely. 


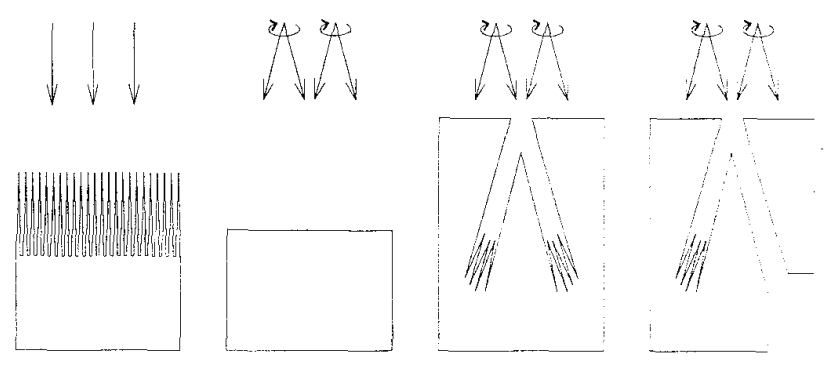

Figure 4: a) The growing and b) preventing of grass. cld) Profile development of a pinhole under a tilted substrate rotation.

Cathode particles: A similar source of particles is the tungsten wire in the Kaufman source which starts the plasma. At this moment we were not able to remove this source without changing the basic idea of IBE.

Ion particles: Another source of particles might be imbedded ions in the PTFE subsurface from the ion source $\left(\mathrm{Ar}\right.$ or $\mathrm{O}_{2}$ ).

Bulk particles: The last source of particles could be the PTFE bulk itself. PTFE always contains a small amount of non-PTFE material sometimes on purpose to give the PTFE a different characteristic like sodium which is difficult to etch. But, even when the PTFE is pure, carbon residue may cause grass forming. During ion sputtering PTFE, gases like $\mathrm{F}_{2}$, $\mathrm{CF}_{4}$, or $\mathrm{C}_{2} \mathrm{~F}_{6}$ are easily created leaving a carbon-rich PTFE surface behind. To be able to remove this carbon overdoses, oxygen ions can be used i.e. RIBE.

\section{EXPERIMENTAL}

Before starting the experiments, the samples have to be cleaned properly, otherwise micromasking will occur. For example, salt from a fingerprint is difficult to etch and will cause irreproducible results. The surface roughness of commercially available PTFE is not good enough to pattern micron sized structures. So, it is necessary to polish the PTFE surface. Then, in most cases, a mask has to be placed on top of the PTFE. Because of the extremely low surface free energy of PTFE this is not straightforward. When the mask is applied on top of the clean and polished PTFE, the sample can be etched for e.g. MEMS applications.

Sample cleaning: PTFE samples to be etched were ordinary bulk material ( $1-10 \mathrm{~mm}$ thick) as well as PTFE foils (50-500micron thick) [23]. To clean the PTFE surface a diluted piranha mixture at $80^{\circ} \mathrm{C}$ was used, i.e. $100 \mathrm{ml}$ of sulphuric acid $\left(\mathrm{H}_{2} \mathrm{SO}_{4}\right)$ gently poured into $500 \mathrm{ml}$ of demiwater and mixed with $100 \mathrm{ml}$ hydrogen peroxide $\left(\mathrm{H}_{2} \mathrm{O}_{2}\right)$. After this treatment the PTFE surface becomes nicely white.

Sample polishing: To create a smooth surface, some samples were polished with silica or alumna balls $(\varnothing \sim 50 \mathrm{~nm})$. After this treatment, the PTFE-surface becomes highly reflective i.e. mirror like. Eventually, the PTFE samples can be cleaned again to remove any polish-residue.

Sample patterning: In order to copy a pattern into the sample, the first problem is the extremely low free energy of the PTFE surface. Therefore direct patterning with photoresist is difficult. Techniques to overcome this problem are: 1) Roughening the PTFE surface with a plasma before spinning resist. 2) The deposition of an intermediate layer such as evaporated aluminium or titanium. 3) The use of a shadow mask e.g. fabricated in silicon [24].

1) Roughening the surface to increase the adhesion for resist seems to be rather clumsy after the polishing treatment and will not be considered.

2) The deposition of an intermediate layer is an excellent way to pattern the PTFE. However, care should be taken in this step. We studied two different metals, aluminium and titanium from which titanium has the lowest etch rate, thus highest selectivity in IBE. To copy the resist pattern into the metal we experienced that wet etching is almost impossible because the layer is stripped. After all, the layer has a bad adhesion with the PTFE. Therefore, the whole etch process should be dry using e.g. RIE. Aluminium can be etched with the help of chlorine-based chemistry and titanium with fluorine-based chemistry. During deposition of titanium with the help of an evaporator, the titanium particles leaving the melt are extremely hot and will dig themselves into the PTFE subsurface [49]. Although this effect will increase the adhesion of the titanium to the PTFE, after patterning the mask with resist it can be difficult to remove the subsurface titanium particles from the spots which should be etched. In contradiction, aluminium which has a low melting point can be evaporated at a minimum deposition rate to prevent aluminium to enter the surface of the PTFE.

3) The use of a shadow mask is a rather old technique, but for our purposes it has a tremendous potential. In this technique, a thin membrane with the pattern is placed in between the ion beam and the sample to be etched. If properly cooled, the mask will etch slowly whereas the heated sample is etched at high speed. Therefore many samples can be etched with the help of the same shadow mask. Even better, the mask can be "refreshed" many times just by depositing a new layer in e.g. an e-beam evaporator. An advantage of this technique is the lag of subsurface particle contamination such as titanium because the surfaces to be etched are $100 \%$ clean.

Evidently, it is possible to create a shadow mask with holes but impossible to create a mask with "open" structures such as pillars. Therefore, in order to create any kind of open structure; i) two different shadow masks are needed, ii) the same mask is used twice but the second time it is rotated by a certain angle, or iii) it has to be used indirectly: A metal layer is evaporated through the shadow mask on top of the PTFE substrate and then the PTFE is etched with this metal as a mask. However, it should be remembered that the metal film is fading near the pattern etches. Information about this subject is found in ref.24.

Sample etching: The prepared PTFE samples were placed downstream the ion source on top of the substrate holder and bombarded with e.g. $1000 \mathrm{eV}$ and $1 \mathrm{~mA} / \mathrm{cm}^{2}$ argon ions and examined with SEM afterwards. Vacuum grease was used to provide a better thermal contact between the sample and the sample holder. Ion incidence was normal to the target 


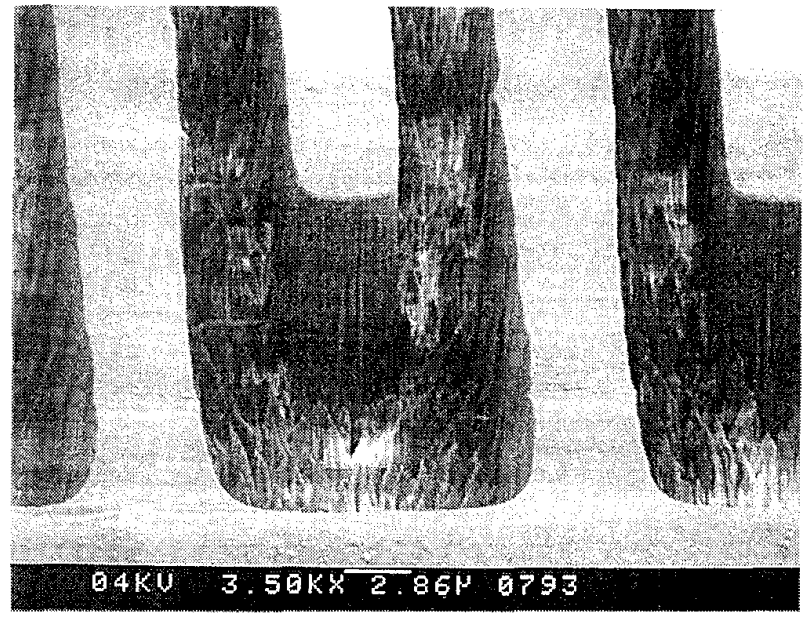

Figure 5: The use of a titanium intermediate layer to transfer a resist pattern into PTFE.

surface except when substrate rotation was used. The vacuum facility pressure were in the $0.1 \mathrm{~m}$ Torr range during ion beam sputtering. However, because of the high etch rate of the FC polymer the pressure near the etching sample can be much higher. To increase the selectivity between a metal mask and the PTFE even further, an oxygen source is used (i.e. TARIBE) which should remove carbon residue also. During etching PTFE samples with argon and oxygen at similar conditions there is no difference in etch rate found. However, oxygen should be able to transform e.g. titanium into the much harder to etch titaniumoxide.

\section{RESULTS \& APPLICATIONS}

Work pioneered by NASA Lewis Research Centre has led to the non-propulsive use of ion thrusters in the area of FC surface modification for biomedical applications including sputter texturing for soft tissue implants $[27,28]$, cardiovascular prostheses [29], percutaneous connectors [30,31], and hydrocephalic shunts [32]. Thin polymer sheets may be ion beam textured and subsequently epoxy bonded to surfaces for moisture protection, load bearing surfaces, or chemically inert low friction surfaces [33-35]. The IBEtextured FC polymers enable the capability of writing or printing on the PTFE surface, decal applications, bonding using adhesive tapes, or as encapsulants for solar cells using silicone adhesives [36]. In literature, additional information is found concerning the roughening and subsequently bonding of non-PTFE surfaces like graphite, surgical stainless steel, titanium, titanium 6-4 nickel, Co-Cr 46-20, CTFE, PFA, FEP, polyurethane, and polyolefins $[36,43]$. These materials are found in the field of biomedicine and implantology e.g. dental implants, orthopaedic and vascular prosthesis, and artificial heart components.

Adhesion: Many methods have been studied to improve the strength of adhesive bonds to fluoropolymers, including chemical treatment [37], rf sputtering processes [21,25,38], glow discharge [39], metal evaporation [40], and others [41]. Among these methods, the sodium etching method has been adapted industrially. The extremely rough surface morphology of sputter textured FC polymers enables strong

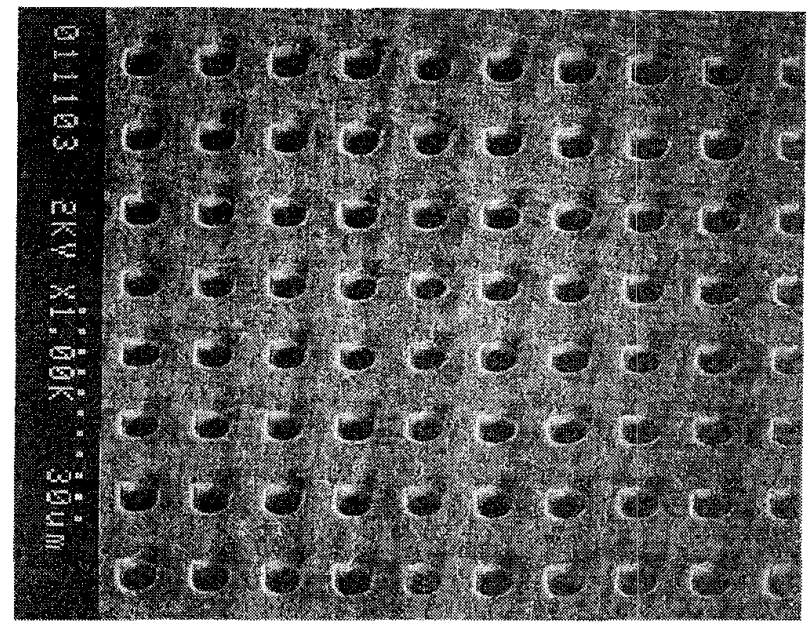

Figure 6: A Teflon sieve with holes of 5 micron etched with the help of a 1 micron thick nitride shadow mask sieve.

mechanical attachment to high modules adhesives. Sputter etched PTFE has demonstrated superior adhesiveness to epoxy resins in comparison to conventional sodium/ naphthalene (Matheson's Poly Etch) treated PTFE $[1,21,25,36]$. The bond can be even stronger when using both techniques subsequently. The use of the intermediate adhesive layer technique to transfer a resist pattern is shown in figure 5 . After evaporating a thin titanium layer on top of a PTFE sheet, resist is spun on top of this layer and RIE patterned with a line pattern. After this, the PTFE is etched in the IBE

Biomaterial: The etched PTFE sheet can be used directly as a bio compatible filter for e.g. blood filtering [26]. Such filters are strong because of the high plasticity of PTFE. In figure 6 an example of a 5 micron filter is given.

Direct moulding: An even more exciting applications is the use of an etched PTFE substrate for direct moulding [11]. Because of the low surface free energy of PTFE, it should be easy to remove an electrochemically grown structure from the PTFE. However, at this moment we were not able to prevent the micrograss completely $(\Rightarrow$ adhesion!), because the IBE system features carbon grids for ion extraction. Figure 7 shows a 55 micron deep structure into a PTFE sheet. The structure is etched with the help of a shadow mask within 30 minutes IBE. At this moment the use of electrons instead of ions to etch PTFE is studied i.e. TAEBE because in this mechanism it is much easier to control and minimise the particle contamination. Moreover, the TAEBE equipment is straightforward and astonishing simple. First results are quite promising because the grass problem disappeared and will be presented in the future.

Microvalve: If substrate rotation is used under a tilted angle, a unidirectional valve will be etched directly under a pin-hole as shown in figure $4 \mathrm{c}$. A flow coming from the pinhole side may easily pass the microvalve whereas a flow from the opposite direction will be blocked.

3D-structuring: Another interesting possibility is to use a PTFE sheet to structure surfaces which are not flat, because the PTFE is easily folded around the structure. To give another fantastic example, it is possible to IBE holes $(\varnothing=18 \mu \mathrm{m})$ into a PTFE tube $(\varnothing=375-625 \mu \mathrm{m})$ using 

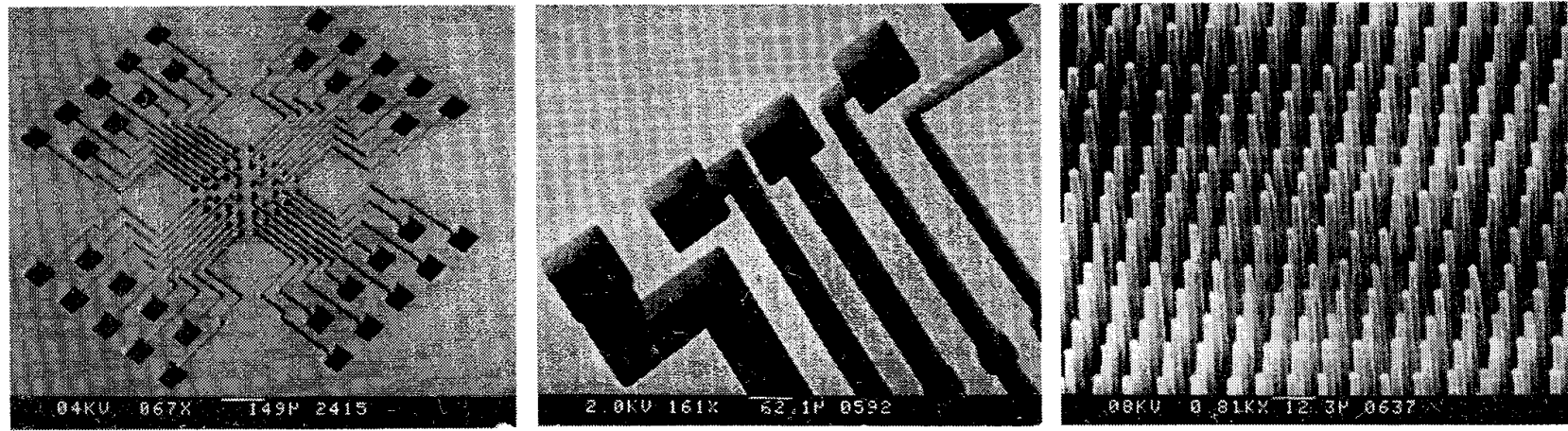

Figure 7a) A 50 micron thick silicon shadow mask with 10 micron resolution, b) A Teflon sheet of 55 microns thick etched with the help of a shadow mask. Figure 8: Pillars 55 micron high, 3 micron in width etched with the reverse shadow mask technique.

electroformed nickel mesh masks for hydrocephalic shunts as demonstrated by Garner [13]. Holes with the smoothest walls and the sharpest definition were obtained by using low beam power densities and a tubing target temperature of less than $50^{\circ} \mathrm{C}$. The perforated microtubules have an important application in medicine for sufferers of hydrocephalus, a malady which results in the build-up of cerebrospinal fluid in the brain. The tubing is inserted into the ventricle and serves as a shunt by draining off the excess cerebrospinal fluid into another part of the brain, where the fluid is absorbed by normal processes. Figure 8 shows an example of the indirect technique to construct 3D structures. It shows PTFE pillars 3 microns in diameter and 55 microns high after 30 minutes IBE. The evaporated mask is $200 \mathrm{~nm}$ of titanium.

Bonding: FC polymers can be first textured and then bonded to themselves using epoxy resin [36,42]. We experienced that a PTFE sheet with an evaporated titanium/ aluminium sandwich layer can be bonded to a glass or prepared silicon wafer. This technique opens a complete new set of possibilities.

MEMS: Etched PTFE structures with deposited metal layers can be used to construct MEMS. Many techniques are available as found in ref.46.

\section{CONCLUSIONS}

It has been concluded that the combination of the unique properties, deposition and etching techniques should make FC thin-films a promising tool for micromachining. A new technique is demonstrated to etch PTFE substrates with high aspect ratios for MEMS applications; thermally assisted ion beam etching (TAIBE). Two important characteristics of TAIBE of PTFE are the extremely high sputter etch rates and the resulting large cone or grasslike surface microstructures. A model is proposed which explains these characteristics: Due to the low thermal conductivity of PTFE and the highly energetic ions the surface temperature thus the vapour pressure increases markedly. Because of the low partial back pressure of vacuum systems the PTFE is not in equilibrium enabling particles to leave the solid. So, incoming ions can transport many molecules into the vapour phase only because they cause a temperature spike at the PTFE surface and the yield is correspondingly high. Many applications are discussed to show its potential.

\section{REFERENCES}

[1] B A Banks, JS Sovey, T B Miller, and K.S.Crandall, NASA TM-78888, 1978 [2] H. Jansen, H. Gardeniers and J. Fluitman, A survey on the reactive ion etching... MME '95, Copenhagen

[3] A.J.Weigand, NASA TM-78851, 1978

[4] H.V.Jansen, J.G.E.Gardeniers, J.Elders, H.A.C.Tilmans, and M.Elwenspoek. S.\&A. A, $41-42,1994$

[5] J.Elders, H.V.Jansen, and M.Elwenspoek, conf. proc. MEMS, Oiso Japan, 1994

[6] W.M. Moreau, Semiconductor Lithography, New York, 1991.

[7] Lehr H. and Ehrfeld W., Aix-en-Provence, France, 1994

[8] J. Moran and D. Maydan, J. Vac. Sci. Tech., 19, 1981.

[9] L. Fried, J. Lechaton, P. Totta, J. Logan, J. Havas and G. Paal, IBM J. Res. Dev 26,1982 .

[10] IJ.W. Bartha, J. Greschner, M. puech and P. Maquin, Microelectronic Engineering, 27, 1995.

[11] O.Auciello, J.Vac.Sci.Tech., 19(4), 1981

[12] J.S.Sovey, J.Vac.Sci.Tech., 16(2), 1979

[13] C.E.Garner, S.B Gabriel, and Y.S.Kuo, Thin Solid Films, 95 (1982).

[14] M. White, Thin solid films, 18, 157 (1973).

15] E.Mathias and G.H.Miller, J.Phys.Chem., 71, 2671 (1967).

[16] D.F.Hall and H.E.Green, AIAA paper No. 72-446, 1972

[17] M.Rost, H.J.Erlet, H.Giegengack, O.Fieder, C.Weissmontel, Thin solid films, 20, 1974

[18] G.K.Wehner, phys.rev., 102, 1956.

[19] P.K Rol, J.M.Fluit, and J.Kistemaker, Physica, 26, 1960

[20] N.Laegreid and G.K.Wehner, J.Appl.Phys.., 32, 1961.

[21] T.Moriuchi, S.Yamamoto, M.Ezoe, H.Tabata, K.Uemori, F.Shigeta, Y.Ohya, A.Tsumuro, and J.Nakai, 7th intern. vac.congr. and 3 rd intern. conf.solid surfaces, Vienna, 1977 .

[22] H. Jansen, M. de Boer, R. Legtenberg and M. Elwenspoek, J. Micromech. Microeng. 5, 1995

[23] Goodfellow Cambridge Ltd

[24] G.J.Burger et al., High resolution shadow mask..., Transducers '95, Sweden.

[25] J.Nakai and K.Fukunaga, US patent No. 4, 064, 030, 1977.

[26] C.J.M. van Rijn and M. Elwenspoek, Micro filtration membrane sieve..., IEEE MEMS 1995., Amsterdam

[27] D.F.Gibbons, NASA contract Rep. CR-159358, 1980

[28] D.F.Gibbons, NASA contract NAS3-22443, 1980

[29] B. A Barks A.JWeigand, C.A.Babbush, and C.L.van Kampen, NASA Tech.Memo TMX-73512, 1976

[30] J.Miller and C.E.Brooks, J.Biomed.Mater.Res., Symp.2, 1971

[31] G.J.Picha and D.F.Gibbons, NASA contract Rep. CR-165255, 1981

[32] E.Foltz, NASA contract NAS3-21963, 1980

[33] J.R.Hollahan, T.Wydeven, and C.C.Johnson, Appl.Opt. 13, 1974.

[34] D. T.Morrison and T.Robertson, Thin solid films 15, 1973.

[35] R.Harop and P.J.Harrop, Thin solid films 3, 1969.

[36] M.J.Mirtich and J.S.Sovey, J.Vac.Sci.T., 16(2), 1979.

[37] A.A.Benderly, J.Appl.Polym.Sci., 6, 221, 1962.

[38] D.M.Matton and J.E.McDonald, J.Appl.Phys. 34, 1963.

[39] H.Schonhon and R.H.Hansen, J.Appl.Polym. Sci., 11, 1967.

[40] R.F.Roberts and F.W.Ryan, H.Schonhom, G.M.Sessler, and J.E.West,

J.Appl.Polym.Sci., 20, 1976

[41] G.C.S.Collins, A.C.Lowe, and D.Nicholas, Eur.Pol.J., 9, 1973.

[42] TRA-CON, Inc. TRA-CAST BA-2114 epoxy resin.

[43] A.J Weigand and B A.Banks, J Vac Sci T Vol 14, No 1, 1977

[44] H. Jansen, $M$ de Boer and $M$. Elwenspoek, High aspect ratio trench etching for MEMS applications, this proceedings

[45] Cuomo, Rossnagel, Kaufman, Handbook of ion beam processing technology, USA, 1989

[46] $M$. de Boer, H. Jansen and M. Elwenspoek, The black silicon method V: Transducers '95, Sweden

[47] R. Kelly, Surf. Sci., 90, 1979

[48] H.W. Starkweather, Jr, Macromolecules, 19, 1986

[49] C. Chang, Y Kim and A.G. Schrott, J.Vac.Sci.T. A8(4), 1990 